Renner, R., Van Hertem, D. (2016). "Potential of wind farms connected to HVDC grid to provide DC ancillary services".

This is the author's version of an article that has been accepted for publication in IEEE Transactions on Sustainable Energy. Changes are made to this version by the publisher prior to publication.

Print ISSN: $1949-3029$

Online ISSN: 1949-3037

Digital Object Identifier: 10.1109/TSTE.2017.2756916

URL: http://ieeexplore.ieee.org/document/8051082

(C2017 IEEE. Personal use of this material is permitted. Permission from IEEE must be obtained for all other users, including reprinting/ republishing this material for advertising or promotional purposes, creating new collective works for resale or redistribution to servers or lists, or reuse of any copyrighted components of this work in other works. 


\title{
Potential of wind farms connected to HVDC grid to provide DC ancillary services
}

\author{
Robert H. Renner, Member, IEEE, Dirk Van Hertem, Senior Member, IEEE
}

\begin{abstract}
DC grids will require ancillary services to compensate for uncertainties such as disturbances and converter outages. The appropriate equipment behaviour and the sources of these services consequently need to be defined. This paper describes the reaction, activation and provision intervals of ancillary services for DC grids to maintain the DC voltage in a certain range. It also discusses how wind power plants and synchronous generators which are exclusively connected to a DC grid can comply with these requirements. The theoretical descriptions are validated using simulations.
\end{abstract}

Index Terms-DC grids, Reserves, Power Systems, Ancillary Services.

\section{INTRODUCTION}

The definition of ancillary services is typically described in a general manner, not to exclude any potential source of a service which could benefit the transmission system. This is reflected by the definitions of ancillary services by the Federal Energy Regulatory Commission (FERC) [1] and The Union of the Electricity Industry (EURELECTRIC) [2]:

- FERC Those services necessary to support the transmission of electric power from seller to purchaser, given the obligations of control areas and transmitting utilities within those control areas, to maintain reliable operations of the interconnected transmission system. Ancillary services supplied with generation include load following, reactive power-voltage regulation, system protective services, loss compensation service, system control, load dispatch services, and energy imbalance services.

- EURELECTRIC All services procured by the transmission or distribution system operator from system users to enable them to maintain the integrity and stability of the transmission and distribution system as well as power quality (to be able to provide system services see definition). They include i.e. frequency control, voltage control, blackstart capability, grid loss compensation (EURELECTRIC report Ancillary services, 2004).

These definitions are also valid for DC grids and the purpose is therefore to maintain reliable operation (FERC) or maintain integrity and stability as well as power quality (EURELECTRIC). A description of procurement and a basic set of ancillary services in transmission systems with DC grids are given in [3].

DC grids require ancillary services to provide electrical energy of a certain quality to, for instance, maintain the

R.H. Renner and D. Van Hertem are with KU Leuven, Department Electrical Engineering (ESAT), Research group Electa, Kasteelpark Arenberg 10 (PB2445), 3001 Heverlee, Belgium, e-mail: dr.r.h.renner@gmx.de, dirk.vanhertem@esat.kuleuven.be

The Electa research group is a founding member of EnergyVille
DC voltage of the DC grid within set limits. As long as these requirements are limited in scope, it is sufficient to "import" the required ancillary services from connected AC power systems. This is because a relatively small DC grid will create minor interferences in the connected AC power systems. The requirements for additional services to compensate these disturbances are therefore minor as well. In this situation the DC ancillary services are covered by the existing AC ancillary services, as is currently the practice.

The responsibility of ancillary service provision and their sources will form an essential part of the design and operation of the DC grid. Especially when DC grids increase in power transmission level, spatial extension and when different types of connections such as offshore wind power plants and islands are integrated. Part of the DC ancillary services can likely be directly provided by DC equipment. This way limitations caused by the transport of ancillary services are avoided. One option is that wind power plants (WPP) which are exclusively connected to the DC grid, provide DC ancillary services, but other sources are also possible. The ability of WPPs to provide AC ancillary services - for example, frequency containment and restoration reserve, power oscillation damping and reactive power - has extensively been discussed in the literature [4][6]. The possibility that WPPs provide ancillary services to AC grids when connected with HVDC point-to-point or multiterminal DC (MTDC) systems has also been explored [7]. It is also noted in [8] that offshore WPP connected to a HVDC system can participate in DC voltage control. Research also shows that the fluctuating nature of WPP can be reduced [9], [10]. However, the requirements for DC grid reserve provision have not been studied before.

This paper focuses on ancillary services which maintain the DC grid voltage and while respecting the power balance of the entire power system. Consequently, the appropriate power service timings for DC grids and their energy content are described. Furthermore, it is analysed how power services might be covered with synchronous generators, as well as WPP and DC storage. For WPP and synchronous generators, this implies a definition of harmonisation, due to differences in reaction time and power constraints. The final part of this paper presents case studies that confirm the general descriptions.

\section{APPROPRIATE RESERVE BEHAVIOUR}

A multi-step reserve is described in multiple publications, although the name associated with these reserves differs [11][13]. Each of the DC reserve steps should align its behaviour to the corresponding $\mathrm{AC}$ reserve step to guarantee reliability and power quality in DC grids, comparable to that in AC grids. 
For this paper, the multi-step reserve described in [11] is used to define an appropriate behaviour:

\section{- DC buffer energy or virtual inertia}

The system dependent DC buffer energy reacts similar to AC grids inertia, although its energy content is smaller. Additional energy is consequently needed to increase the reaction time after a severe imbalance. Its behaviour is not limited to differential control in the way that inertia is in AC grids. The easiest way to implement it is thus via a droop with no or very small deadband. Its energy content should be as small as possible in order to limit the required amount of storage.

- DC voltage containment reserve (DCVCR)

DCVCR should react as fast as possible and should remain available at least until DC voltage restoration reserve is fully activated. One option is to extend the idea of solidarity, with DC voltage containment reserve supporting AC power systems and vice versa, [13].

- DC voltage restoration reserve (DCVRR)

DCVRR should react within the same time range as its $\mathrm{AC}$ equivalent and it should also have the same availability, [11].

Based on the above descriptions, Figure 1 can be drawn. It illustrates the sequence of DC buffer energy and reserve steps. Further analysis shows the need to define the reaction,

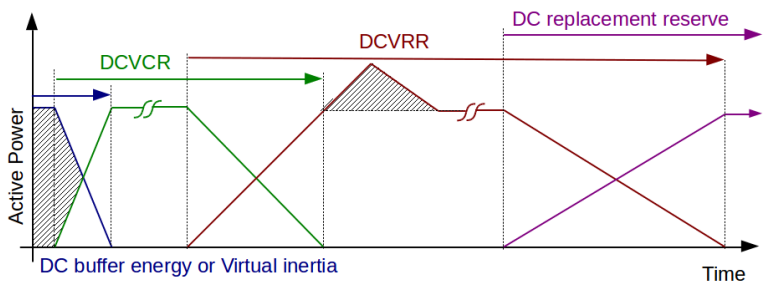

Figure 1: Suggested multi-step reserve for DC grids.

activation and provision interval for each of these DC services.

The buffer energy, $\Delta E_{\mathrm{Cap}}$ and $\Delta E_{\mathrm{Rot}}$, in a DC and $\mathrm{AC}$ system can be calculated by equation (1) and (2) respectively.

$$
\Delta E_{\text {Cap }}=P \cdot t=\frac{1}{2} \cdot u(t)^{2} \cdot C-\frac{1}{2} \cdot u(0)^{2} \cdot C
$$

Where $C$ is the total system capacitance and $t$ the charge or discharge duration with constant power $P$ which results in a voltage change from $u(0)$ to $u(t)$.

$$
\begin{aligned}
\Delta E_{\mathrm{Rot}} & =\frac{1}{2} \cdot J\left(\omega(t)^{2}-\omega(0)^{2}\right) \\
& =\frac{H \cdot S_{\mathrm{r}}}{\omega_{\mathrm{n}}^{2}} \cdot\left(\omega(t)^{2}-\omega(0)^{2}\right)
\end{aligned}
$$

Where $J$ the total moment of inertia, $\omega(t)$ the angular frequency at the time $t, \omega(0)$ the angular frequency at the time 0 , $\omega_{n}$ the nominal angular frequency, $H$ the total inertia constant and $S_{r}$ the total rated apparent power.

The buffer energy, $\Delta E_{\mathrm{Cap}}$ and $\Delta E_{\mathrm{Rot}}$, are system-dependent and increase with the number of HVDC converter stations and synchronous generators respectively, as explained in [11]. The restoration reserve of both transmission systems should moreover have the same activation and provision time range to prevent unwanted disturbances. The remaining intervals follow accordingly when these definitions are used. When $t_{F A}$ is defined as the time until full activation for DCVRR, which should not be longer as the DCVCR is minimally available, the energy content of the DCVCR becomes:

$$
E_{V C R}=t_{F A} \cdot P_{M O u t}
$$

With $E_{V C R}$ the needed energy and $P_{M O u t}$ the maximum power outage.

Usually, control areas do not use uniform definitions for start of deployment nor full activation of FRR. In Europe, for example, the start of deployment ranges from as fast as possible to $30 \mathrm{~s}$ and the full activation from 5 to 15 min [14]. It is therefore assumed that the DCVRR has the fastest possible start of deployment and a full activation time of $t_{F A}=5 \mathrm{~min}$. With these values and a maximum power outage of $P_{\text {MOut }}=1 \mathrm{GW}$, equation (4) results in $E_{V C R}=300 \mathrm{GWs}=83.33 \mathrm{MWh}$. In general, the source of this energy can be freely chosen, but it has to be provided within the required containment reserve activation time.

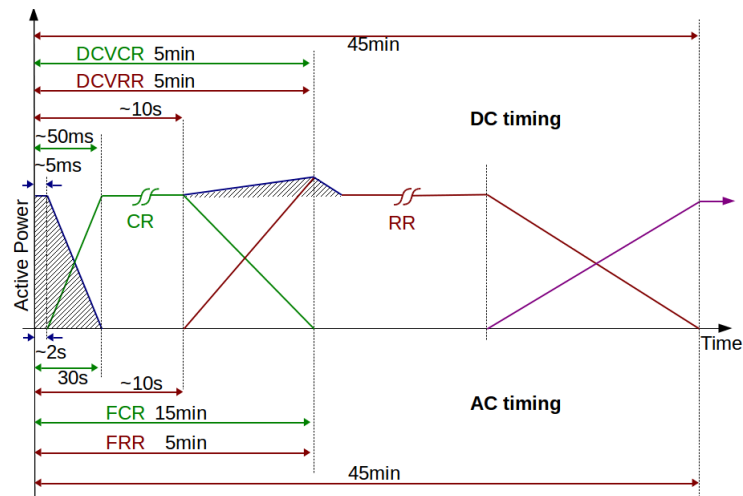

Figure 2: Technical necessary or proposed reaction, activation and provision times of reserves for DC grids and reaction, activation and provision times of reserves for the AC transmission system of continental Europe [14].

The results of the previous analysis are shown in Figure 2 upper half. The shape is the same as for the AC grid shown in Figure 2 lower half, but the defined times differ. The reserveproviding power plants have to meet the shown reaction, activation and provision times. How this can be achieved is discussed in the next section.

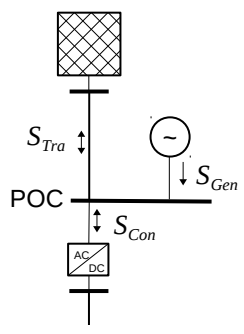

Figure 3: Definition of converter power $S_{C o n}$, generation power $S_{G e n}$ and transmission capacity $S_{T r a}$. 


\section{SOURCES OF DC RESERVES, THEIR POTENTIAL AND CONTROL}

This section discusses the potential sources for DC reserves from conventional generators and wind farms. Both these types of generation can be connected to an $\mathrm{AC}$ island, a weak $\mathrm{AC}$ system or a strong $\mathrm{AC}$ system. For each application, the $\mathrm{AC} / \mathrm{DC}$ converter and the generator consequently require a different grade of coordination with respect to active and reactive power control. An AC island with one generator needs a much higher coordination with the converter than a generator connected to a strong $\mathrm{AC}$ power system. It is consequently useful to define a converter coordination constant $H_{C}$, which depends on the converter power $S_{C o n}$, the generation power $S_{G e n}$ and the transmission capacity $S_{T r a}$ at the point of connection, see Figure 3 for definitions.

$$
H_{C}=\frac{\sum S_{G e n}-\sum S_{T r a}}{\sum S_{C o n}}
$$

If $H_{C}$ is $\leq 1$, the converter has to guarantee that the power $>\sum S_{T r a}$ is exported. For a value $H_{C}>1$, the generators have to be curtailed. If $H_{C}$ is between 0 and -1 , the converter has to coordinate its infeed with the connected generators, and if $H_{C}<-1$, the AC connection can handle the entire generator and infeed converter power. $S_{C o n}$ is defined positive and as the base power for all values. For more complex configurations a more advanced method is needed, as described in [13].

\section{A. Synchronous generator}

If $H_{C}>-1$, the reserve-providing generator and the related VSC need to harmonise their active power generation and transmission. Conventional generation provides a large inertia, represented by the inertia constant, which typically range from $2 \mathrm{~s} \leq H \leq 10 \mathrm{~s}$ [15], and results in slow reaction times. When this type of generation is exclusively connected to an HVDC VSC, the converter control parameters have to correspond to the generator dynamics. The maximum instantaneous power change of the converter is in this case limited by the available inertia of the synchronous generator and its accepted frequency deviation. Equation (3) divided by time therefore has to correspond with that which is activated by the DC droop:

$$
K_{p}=\frac{\Delta P_{P M a x}}{U_{n}-U_{L i m}}
$$

This results in

$$
\Delta P_{P M a x}=\frac{H \cdot S_{r}}{\Delta t \cdot \omega_{n}^{2}} \cdot\left(\omega_{n}^{2}-\omega_{L i m}^{2}\right)=K_{p} \cdot\left(U_{n}-U_{L i m}\right)
$$

With $\Delta t$ the time that power is activated, which is roughly half the activation time of the generator's containment reserve, as shown in Figure 2. This time moreover depends on the generator type and is limited by the grid codes. In Europe, for instance, the grid codes request a maximum activation time of $30 \mathrm{~s}$.

The DCVCR relies on inertia provided by the AC system due to the fundamental differences in activation times. The energy stored in the rotating mass can therefore be used to damp the DC grid. The disadvantage of a high inertia is that the power changes at a slow rate when the set-point is adjusted.
Its maximum rate of change thus needs to be considered if this type of power plant is to provide DCVRR. This rate of change is typically in the range of $4-8 \% / \mathrm{min}$ [16].

For the simulations, conventional power plants are modelled with the integrated DigSilent model and the parameters shown in Table III to V.

\section{B. Wind power plants}

WPPs can contribute to DC ancillary services through de-rating and de-loading. De-rating means that the WPP is permanently operated below its power capability, and that the reaction time can be within seconds. This strategy can guarantee a high power reserve, but calls for a reliable wind forecast and comes at a cost. The power and timing limits are shown in Figure 4.

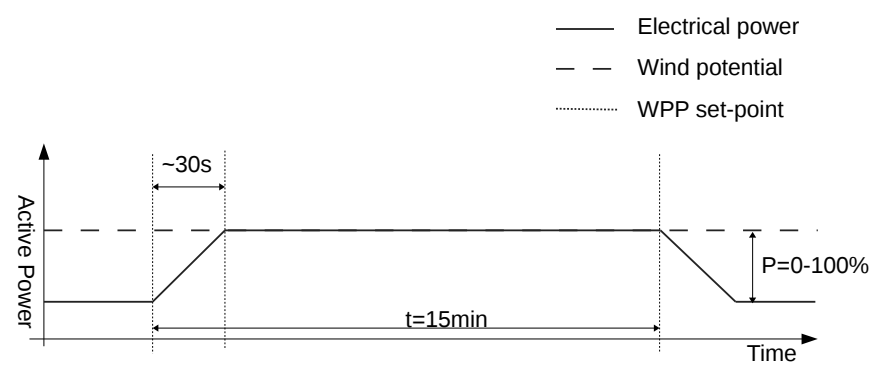

Figure 4: WPP de-rating capability.

De-loading uses the energy stored in the rotating parts of the WPPs. The reaction time is consequently within hundreds of ms, but its power is limited to around 10\% of the WPP's steady state operation point. Availability is moreover limited to some seconds as the rotational speed of the turbine must not deviate more than roughly $10 \%$ from its nominal value, while the withdrawn energy has to be returned afterwards, which produces a rebound effect. This is illustrated in Figure 5.

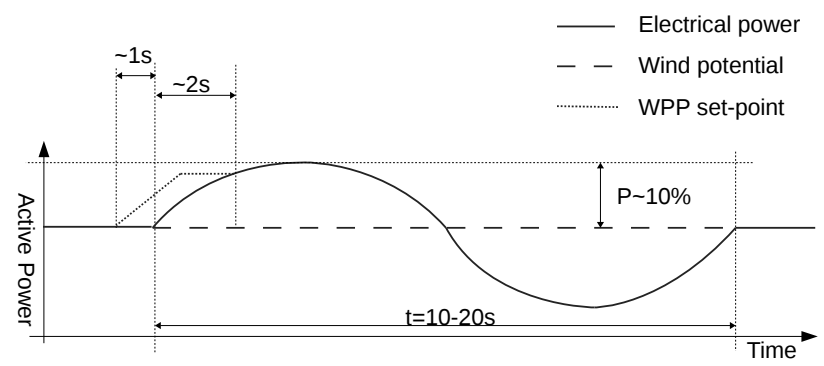

Figure 5: WPP de-loading capability.

Neither strategy is fast enough to support a DC grid with buffer energy, and this makes it necessary to either use DC storage or rely on AC grid inertia. A combined de-loading/derating strategy can provide a DCVCR with the limitations shown in Figure 6, and an assumed provision time of minimally $5 \mathrm{~min}$. From a controller perspective, the provision of DCVRR is also an option. It should be taken into account, however, that a reliable forecast for $45 \mathrm{~min}$ is needed, or that shorter intervals have to be defined. 


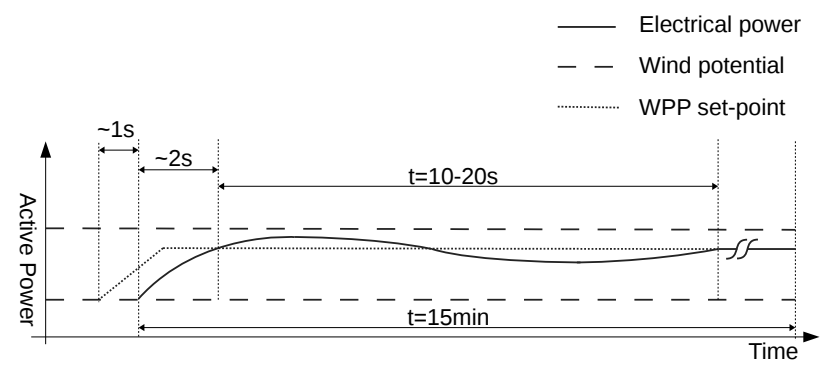

Figure 6: Combined de-loading and de-rating capability.

The provision of DC reserves by WPPs was simulated using the full converter WPP model available in Digsilent [17]. This model has no aerodynamic part, which made it necessary to implement set-point gradient limits and communication delays. Both are necessary to simulate the de-loading and de-rating limits previously described. The time delays are realised by a first-order-plus-dead-time (FOPDT) model commonly used for time delays [18]. Its mathematical description is

$$
A(s)=\frac{1}{1+T_{1} s} e^{-T_{2} s}
$$

with $T_{1}=$ the equivalent time constant of the plant and $T_{2}=$ the equivalent time constant of the delay. For the simulations, $T_{1}=2 s$ and $T_{2}=1 s$ are used.

\section{Converter control}

Two types of converter control are used in this section. The first one is described in [11], used for conventional generators, with the converter defining the active power exchange between the AC and DC system. The imbalance in the AC system is therefore created by the converter and compensated by the generator.

The second converter controller is used when wind turbines are exclusively connected to the VSC. The converter in this case maintains the AC system frequency by controlling the active power and the AC system voltage by controlling the reactive power. This is achieved by replacing the PLL with a constant frequency input and the active power controller with a frequency controller. An overview is shown in Figure 7.

\section{DC grid storage}

DC storage can be used to increase the energy buffer or to provide the entire DCVCR. The decision whether to include additional storage depends on economic considerations. The question is whether it is more beneficial to provide DC reserves from the DC side and thus utilise the converters to a higher degree, or to provide DC reserves from available AC sources. From a theoretical and technical perspective, DC storage is the preferred solution, due to its ability to provide buffer energy and DCVCR without restricting WPP generation or converter transmission capability.

It would moreover be beneficial to have controllable DC storage to charge and dis-charge when desired. In the performed simulations, however, the implementation was simplified by increasing the converter stations' capacitances. The

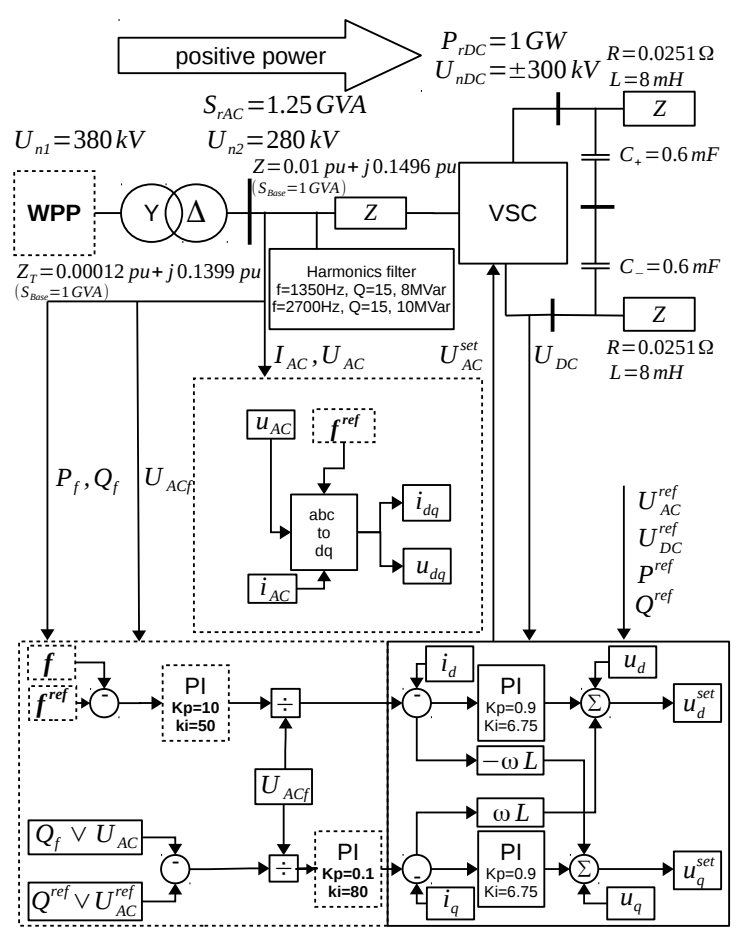

Figure 7: Modified converter control for offshore WPPs. Modifications marked with dashed boxes and changed parameter are written bold.

total capacitances can be calculated with equation (9).

$$
t=\frac{C}{2 \cdot P} \cdot\left(u(t)^{2}-u(0)^{2}\right)
$$

\section{Simulation RESUlts}

For the simulations, a test system with the scheduled DC voltages and power exchange of Figure 8 is defined. This test system consists of four converter stations connected to a meshed grid and split into two control areas, which are connected via two tie lines. Control area north consists of

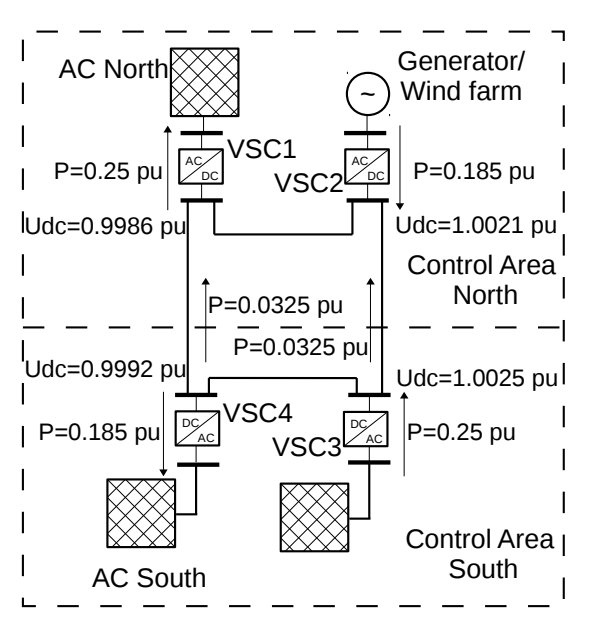

Figure 8: Test Grid. 
$\mathrm{AC}$ grid north and the generator under test, while control area south consists of two converter stations each connected to an $\mathrm{AC}$ grid. Each of the converters has a certain task and the task of VSC2 varies depending on the performed test. The base power is $1000 \mathrm{MW}$ and the base DC voltage is $600 \mathrm{kV}$ for the test grid and the simulations, if not mentioned in particular.

Case studies are performed to investigate the effect of the buffer energy, DCVCR and DCVRR from conventional generation and wind power plants. The contribution of the test generator is limited to the analysed service so as to distinguish between the three types of reserve and their influence on the generator being tested. Therefore, the test cases are split in the Sections "Buffer energy and DCVCR" and "DCVRR", which are showing the response of the related reserve providing controller. The former one has furthermore the paragraphs "Slow Changing Imbalance", "Step Change Imbalance without DC Storage" and "Step Change Imbalance with DC Storage", each paragraph analysing the described disturbance.

\section{A. Buffer energy and DCVCR}

As the buffer energy in the DC grid is limited, even small power deviations result in large DC voltage changes [11]. A permanent DC voltage droop stabilises the DC grid and reduces such changes [19]. This is described by:

$$
\Delta P_{d c}=\frac{1}{k_{d c}} \cdot \Delta U_{d c}=K_{d c} \cdot \Delta U_{d c}
$$

With $\Delta P_{d c}$ the power imbalance, $\Delta U_{d c}$ the DC voltage change, $\frac{1}{k_{d c}}$ the droop and $K_{d c}$ the proportional gain. The permanent DC voltage droop is designed to limit the maximum DC voltage deviation to $\pm 5.5 \%$ for a sudden power change of $\Delta P= \pm 0.25 \mathrm{pu}$. The result is a proportional gain for

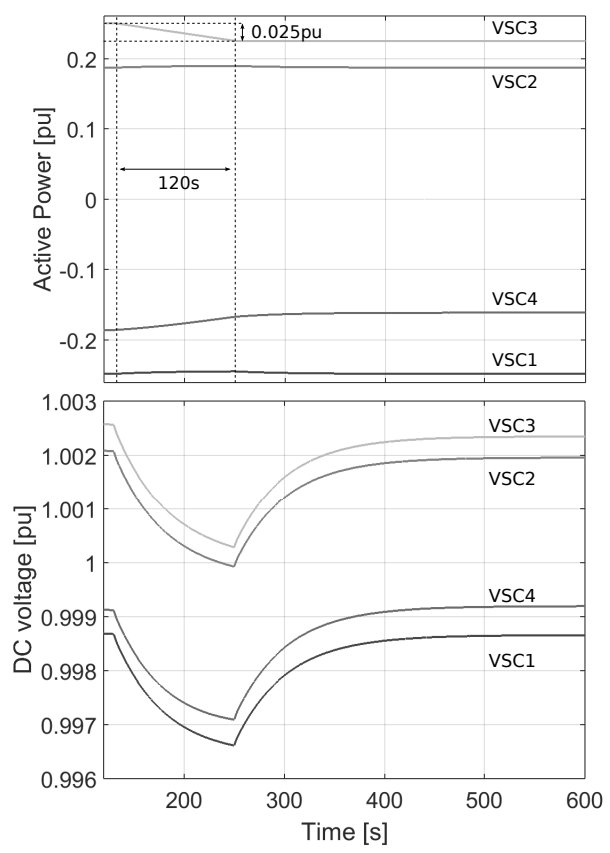

Figure 9: Active power of the converters and DC voltage at converter nodes for the case study: Synchronous generator with slow imbalance change. the DC grid of $\approx 7,58 \frac{M W}{k V}$. The maximum contribution of the synchronous generator is moreover limited by equation (7). For the described generator/converter combinations, this results in:

$$
\begin{aligned}
P_{P M a x} & =4 \cdot \frac{255 \mathrm{MW} \cdot 5 \mathrm{~s} \cdot\left((50 \mathrm{~Hz})^{2}-(49.2 \mathrm{~Hz})^{2}\right)}{(50 \mathrm{~Hz})^{2} \cdot 2.5 \mathrm{~s}} \\
& =64.76 \mathrm{MW} \\
\Rightarrow K_{P} & =\frac{64.76 \mathrm{MW}}{33 \mathrm{kV}}=1.96 \frac{\mathrm{MW}}{\mathrm{kV}}
\end{aligned}
$$

The proportional gains are thus the same as the ones listed in Table I, with VSC3 the imbalance-creating converter. The values remain the same when a WPP/converter system is tested so as to obtain comparable results. Two scenarios are

Table I: Converter droop settings for the case studies: Buffer energy and DCVCR.

\begin{tabular}{c|c} 
Converter & Proportional gain \\
\hline VSC1 & $2.81 \mathrm{MW} / \mathrm{kV}$ \\
VSC2 & $1.96 \mathrm{MW} / \mathrm{kV}$ \\
VSC3 & $0 \mathrm{MW} / \mathrm{kV}$ \\
VSC4 & $2.81 \mathrm{MW} / \mathrm{kV}$
\end{tabular}

simulated with this configuration to show the behaviour of a synchronous generator/converter system with a harmonisation factor $H_{C}=1$. The first one looks at slow imbalances and damping of the DC grid, while the second one shows the response after a power step.

\section{Slow Changing Imbalance}

Figures 9 to 12 illustrate the results for the defined limitations and a slowly changing imbalance. The tasks of the

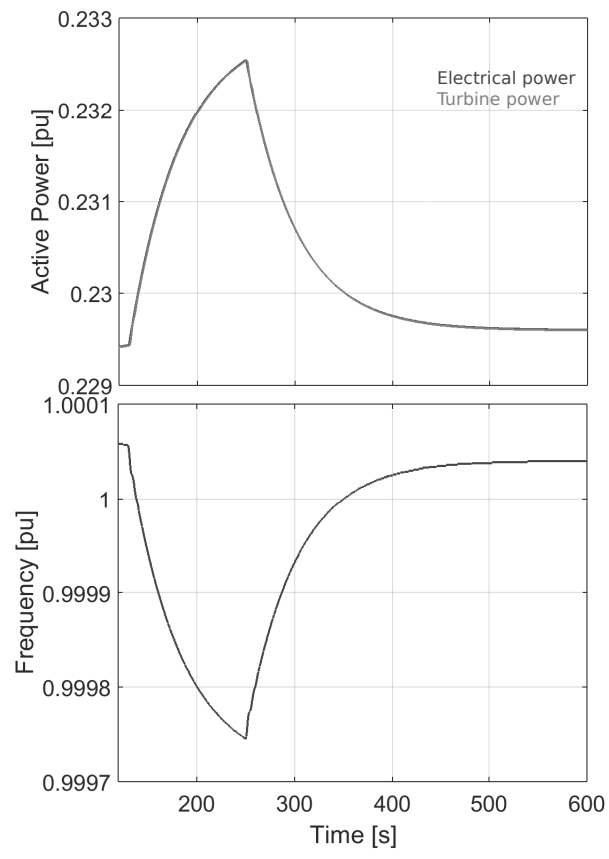

Figure 10: Total active power $($ base power $=816 \mathrm{MW}$ ) and frequency of the synchronous generator for the case study: Synchronous generator with slow imbalance change. 


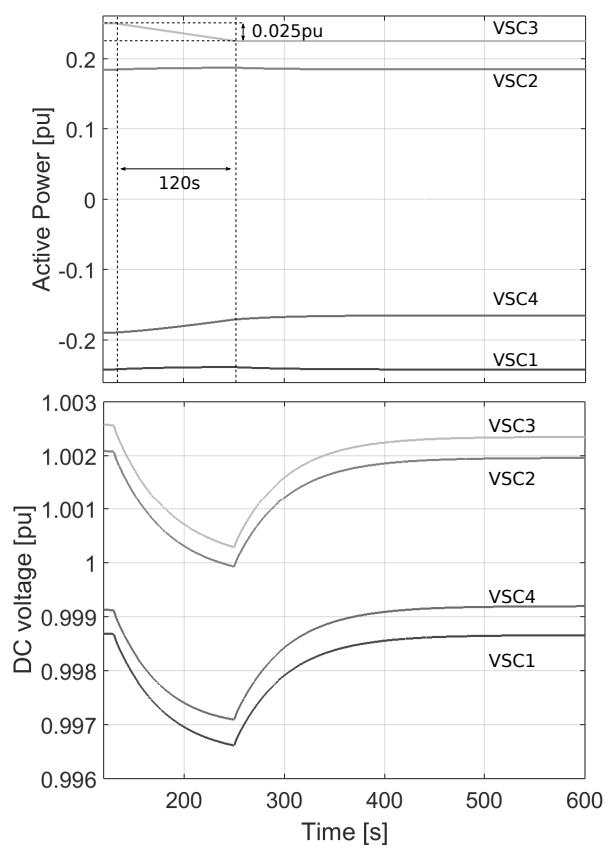

Figure 11: Active power of the converters and DC voltage at converter nodes for the case study: WPP with slow imbalance change.

converter stations are defined as follows: Converter VSC4 is responsible for the DCVRR of control area south, while converter VSC3 slowly changes its active power to create a power imbalance in the DC grid. Most of the imbalance is compensated by the DCVRR, but a small part is transferred via VSC2 and compensated by the frequency droop of the

connected generator. When VSC3 reaches a steadystate, the total imbalance is compensated by VSC4. Every imbalance too fast to be compensated by the DCVRR is thus compensated by the converters providing DC voltage droop. The DC voltage consequently slightly changes as shown in Figure 9, but it is re-established when the disturbance stops. From the perspective of the synchronous generator, this results in a frequency deviation with the same shape as the DC voltage. The frequency deviation is subsequently compensated by the generator governor, see Figure 10. Similar results are obtained for the WPPs, when they are operated in de-rated mode. The slow power change of VSC3 is mainly compensated by VSC4, which is responsible for the DCVRR of control area south. The part not compensated by VSC4 is compensated by the DC voltage droop, and therefore shifted to the WPP, as shown in Figure 11. Due to the control scheme, with VSC2 controlling the frequency and the WPP the power, the frequency remains almost constant at $1 \mathrm{pu}$, while the WPP power follows the DC voltage, as depicted in Figure 12.

\section{Step Change Imbalance without DC Storage}

In the following case is a power step change, which simulates a converter outage. The DCVRR is deactivated to acquire a better understanding of the influence of the equipment under test. The DC voltage droop settings for VSC1, VSC2 and VSC4 are therefore set as listed in Table I, with VSC3

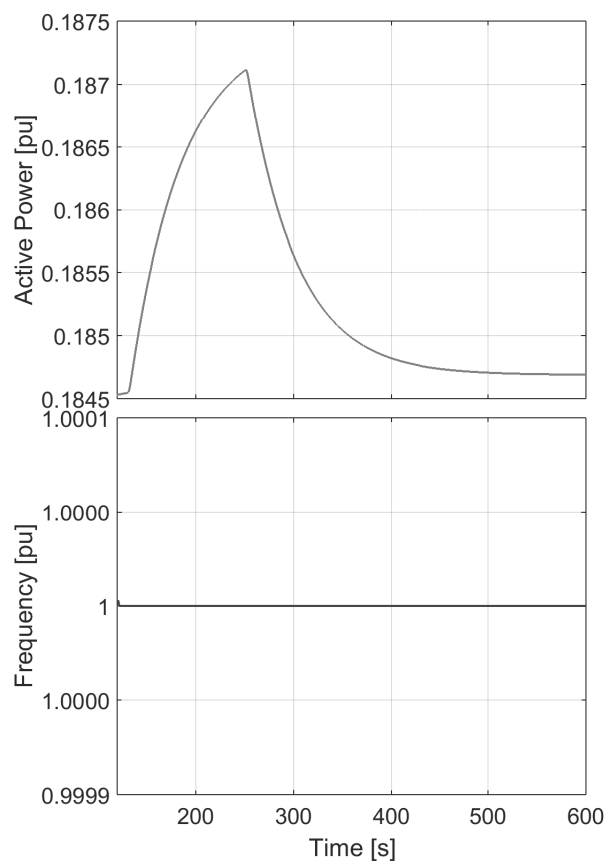

Figure 12: Total active power and frequency of the WPP for the case study: WPP with slow imbalance change.

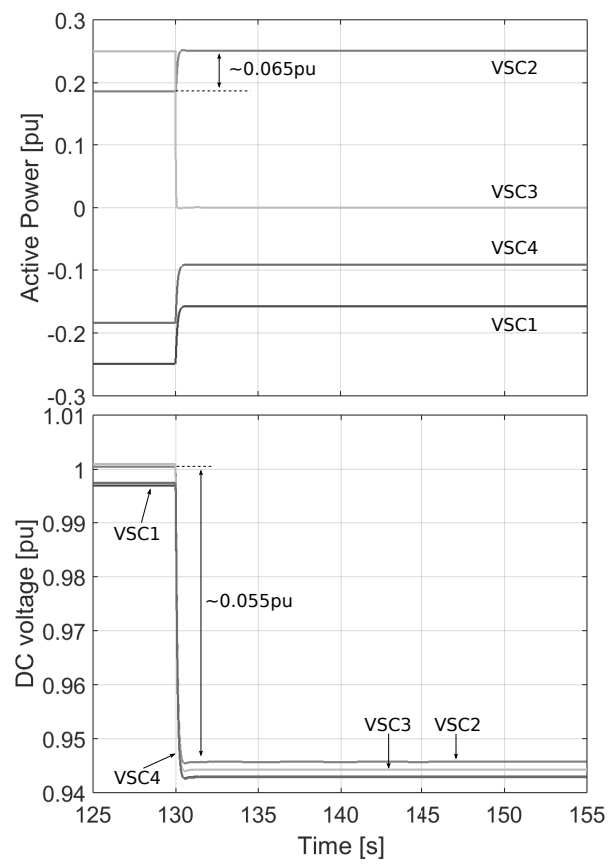

Figure 13: Active power of the converters and DC voltage at converter nodes for the case study: Synchronous generator with power step change.

responsible for the imbalance. This is shown in Figure 13 where the converters adjust their power based on their droop settings. The DC voltage is stabilised at around $0.945 \mathrm{pu}$.

The synchronous generator's response to this sudden power change is depicted in Figure 14. The electrical power output instantaneously increases, albeit in two phases. First, the inertia of the synchronous generator provides the power, which 
results in a maximum frequency decrease of about $0.984 \mathrm{pu}$. The turbine then increases its power output. This process lasts a couple of seconds as the turbine's steam flow has to change. After roughly 20 seconds, the turbine provides the total power and the frequency is stabilised at around $0.9925 \mathrm{pu}$. This value depends on the governor settings, listed in Table V.

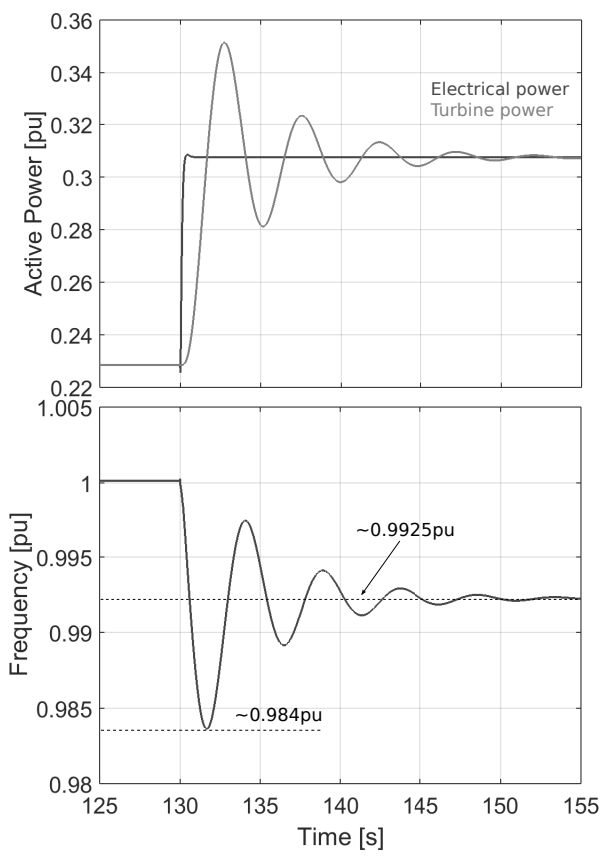

Figure 14: Total active power (base power $=816 \mathrm{MW}$ ) and frequency of the synchronous generator for the case study: Synchronous generator with power step change.

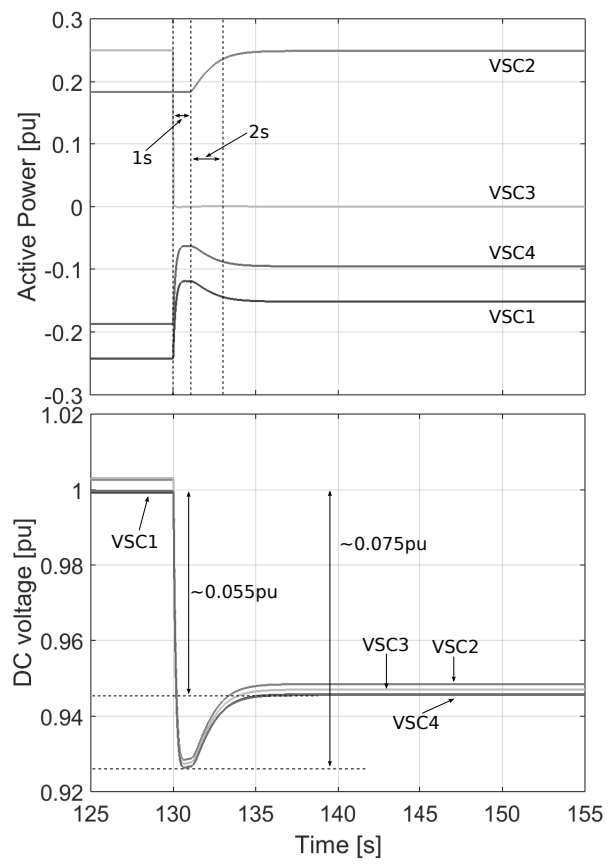

Figure 15: Active power of the converters and DC voltage at converter nodes for the case study: WPP with power step change.
When the same test is performed with a WPP, a deeper DC voltage dip occurs due to the reaction time restrictions. This is shown in Figure 15, where VSC1 and VSC4 compensate the total imbalance, while VSC2 starts to compensate its part $1 \mathrm{~s}$ delayed, and it takes $5 \mathrm{~s}$ for the total power to be activated. As shown in Figure 16, the power of VSC2 and the WPP is almost identical, and the power from DC grid capacitances is activated for only a limited amount of time. Due to the fast reaction time of the converter station, the frequency deviation is both short in time and small in scope.

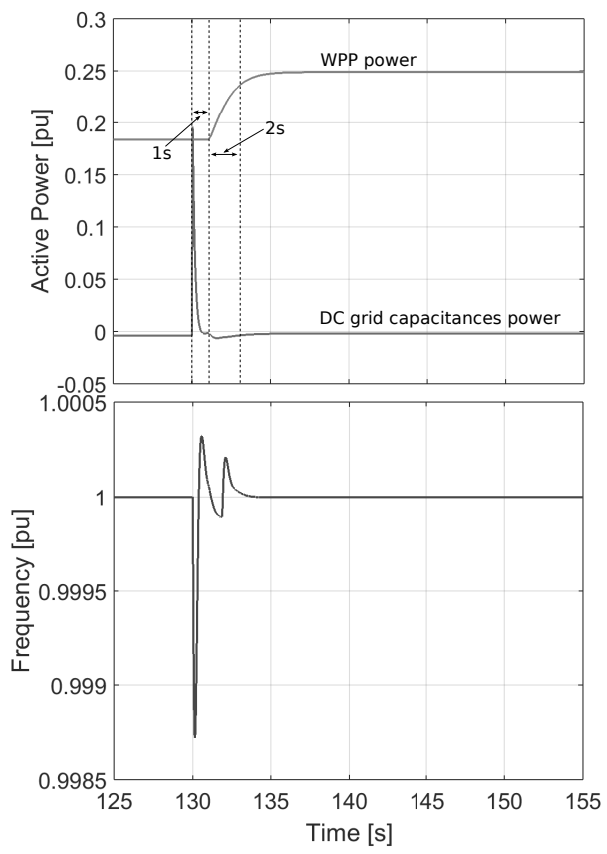

Figure 16: Total active power and frequency of the WPP for the case study: WPP with power step change.

\section{Step Change Imbalance with DC Storage}

Storage can provide the buffer energy if VSC1 and VSC4 have no spare capacity to provide the VSC2 reserve, even for a short time. Due to the simplicity of the modelling approach, this is illustrated in the presented simulations by increasing the capacitances of the converter stations. Equation (9) is used to define the needed capacitance, with a total time of $2 \mathrm{~s}$. This time is estimated with $T_{1}+T_{2} / 2$, where $T_{1}$ and $T_{2}$ are from equation (8). The linearisation ensures an overestimation of the energy storage. The result is a total DC grid capacitance of

$$
C=\frac{1000 M W \cdot 2 s \cdot 2}{\left.(632.1 k V)^{2}-(600 k V)^{2}\right)} \approx 0.1 F
$$

With this capacitance, the power flows through VSC1 and VSC4 do not exceed their defined values, while the DC voltage remains within the defined range. This is shown in Figure 17. The power of the WPP and VSC2 are moreover almost identical.

However, the power from the storage in this case remains activated for a longer time and it compensates the WPP's delay. This is illustrated in Figure 19. Due to the WPP's slow power change, the frequency deviation is insignificant and the spike at $\mathrm{t}=134 \mathrm{~s}$ is a numerical glitch. 


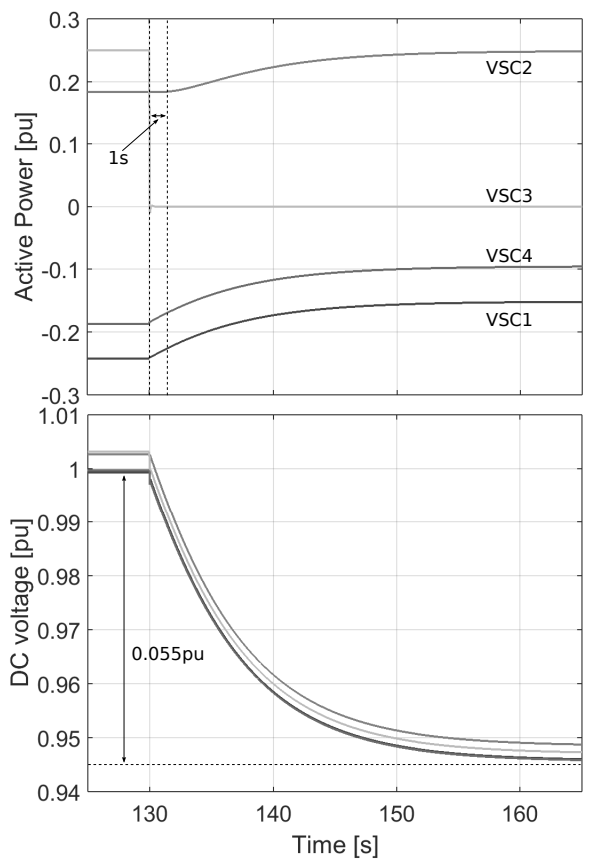

Figure 17: Active power of the converters and DC voltage at converter nodes for the case study: WPP with power step change and additional DC storage.

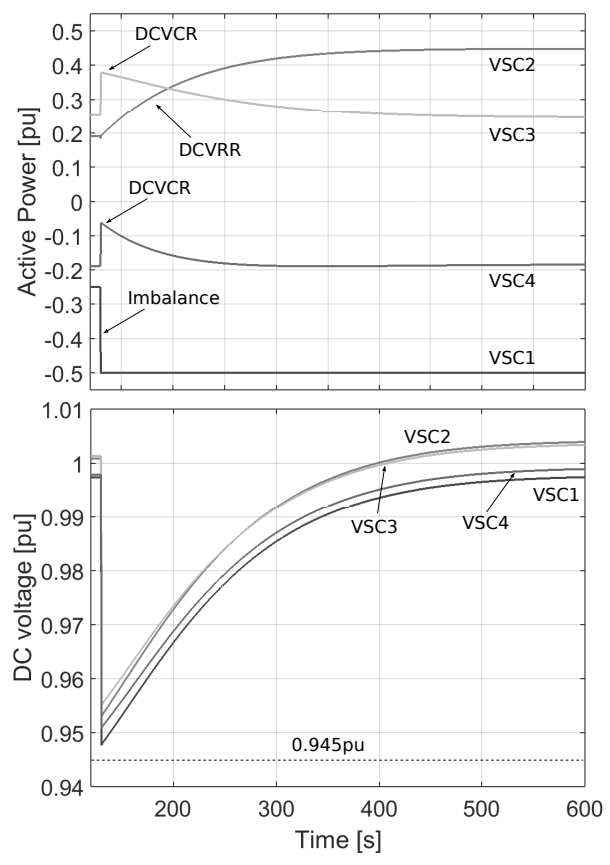

Figure 18: Active power of the converters and DC voltage at converter nodes for the case study: Synchronous generator DCVRR.

\section{B. DCVRR}

The capability of synchronous generators and WPPs to compensate small or slow changes was already shown in the previous section. Consequently, the simulations in this Section are limited to a power step change of $\Delta P=0.25 \mathrm{pu}$. The converters' responsibility and the $\mathrm{DC}$ voltage proportional gains

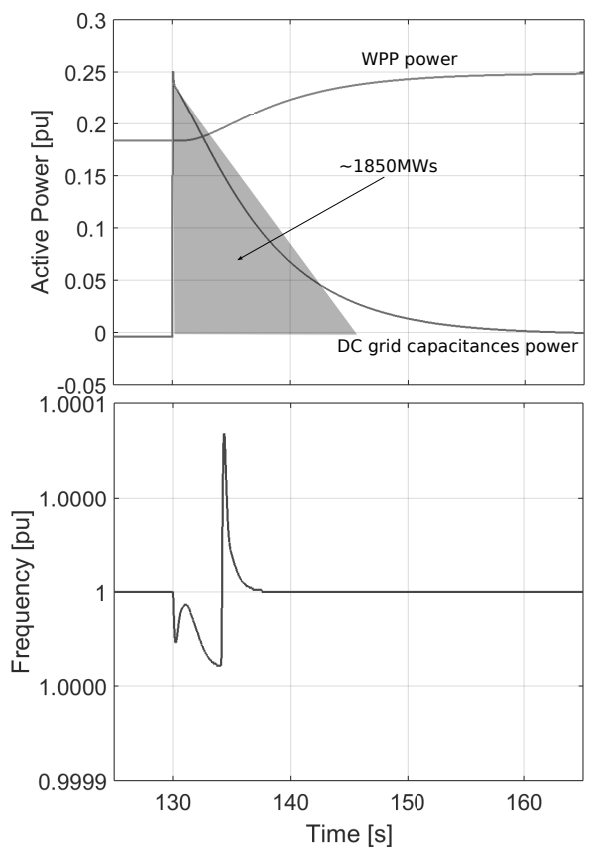

Figure 19: Total active power of the WPP and frequency for the case study: WPP with power step change and additional DC storage.

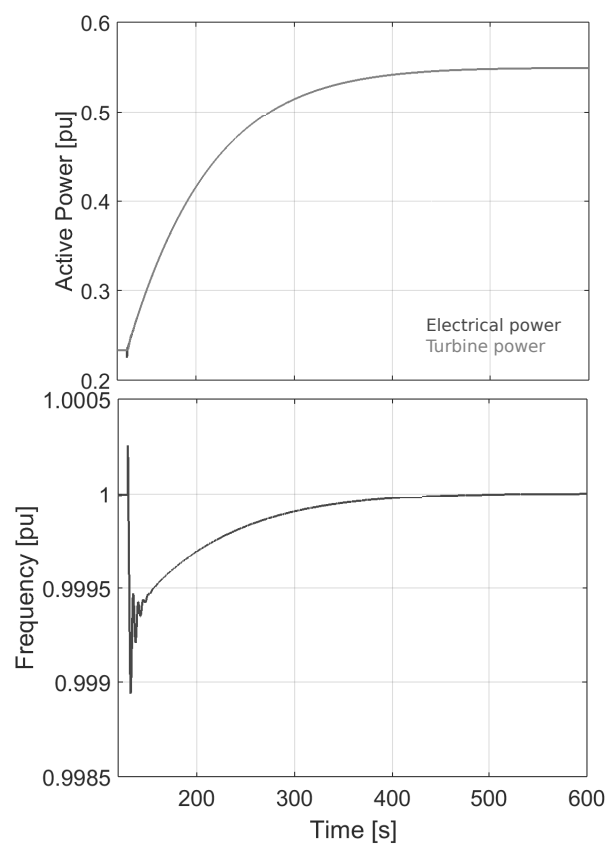

Figure 20: Total active power (base power $=816 \mathrm{MW}$ ) and frequency of the synchronous generator for the case study: Synchronous generator DCVRR.

are adapted to explicit show the DCVRR control reaction. VSC3 and VSC4 are thus responsible for DCVCR, while VSC1 creates an imbalance and VSC2 is responsible for the DCVRR. An overview of the proportional gains is given in Table II. Their sum is designed in the same way as in the previous section. An outage of $\Delta P=0.25$ pu consequently results in a DC voltage change of $\pm 5.5 \%$. Figure 18 illustrates 
Table II: Converter droop settings for the case study: DCVRR.

\begin{tabular}{c|c} 
Converter & Proportional gain \\
\hline VSC1 & $0.00 \mathrm{MW} / \mathrm{kV}$ \\
VSC2 & $0.00 \mathrm{MW} / \mathrm{kV}$ \\
VSC3 & $3.79 \mathrm{MW} / \mathrm{kV}$ \\
VSC4 & $3.79 \mathrm{MW} / \mathrm{kV}$
\end{tabular}

the converters' active power and DC voltage at their DC nodes. Converter VSC1 changes its set-point from $0.25 \mathrm{pu}$ to $-0.5 \mathrm{pu}$, which results in a DC voltage decrease halted by converter VSC3 and VSC4. VSC2 subsequently increases its power to compensate the DCVCR and restore the DC voltage of VSC1 to the scheduled value. Figure 20 shows that the power of the synchronous generator exactly follows the power of VSC2, because the controller setting of the DCVRR is designed to respect its maximum rate of change. The frequency initially decreases slightly and it recovers after some seconds. For WPP, the results look the same. The DC grid is stabilised by the droop-providing converters VSC3 and VSC4, and is subsequently brought back to a defined situation by the DCVRR, figure 21. The power of the WPP follows VSC2, and the frequency deviation is quickly compensated, figure 22 .

It should be noted, however, that this service can only be provided by WPPs when the forecast is reliable for the defined provision time and when it is economic to operate the WPP in de-rated mode for this time period.

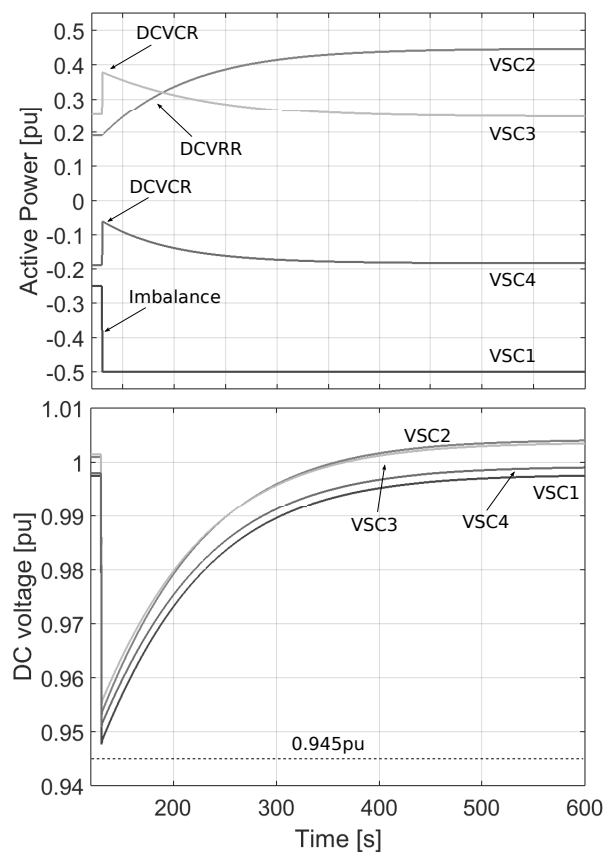

Figure 21: Active power of the converters and DC voltage at converter nodes for the case study: WPP DCVRR.

\section{CONCLUSION}

This paper defined a coordination factor for generation and VSC, as well as describing the behaviour of AC and DC grids at the level of buffer energy, containment reserve and restoration reserve. This paper also illustrates how synchronous

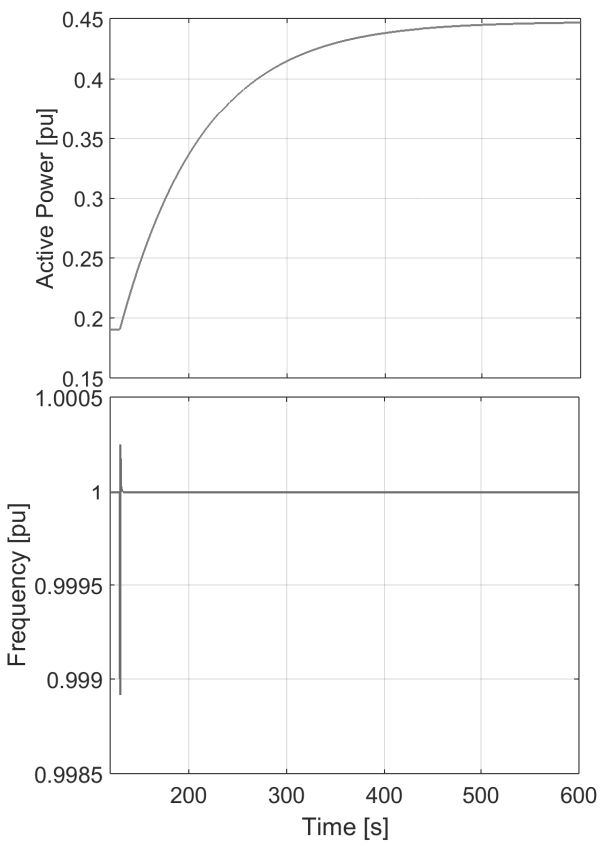

Figure 22: Total active power and frequency of the WPP for the case study: WPP DCVRR.

generators and WPPs with a coordination factor of $H_{C}=1$ can provide energy services to DC grids. Furthermore, a method was presented to calculate the maximum instantaneous power a synchronous generator can provide and how this is related to the proportional gain of the connected VSC. In addition, this paper discussed how adequate storage for WPPs could be defined to handle the same instantaneous power change. The paper concluded with simulations that compared the ability of synchronous generators and WPPs to provide DC containment and DC restoration reserve. This paper consequently presents how ancillary services for DC grids can be delivered in practice, and similarly to current practice. As a next step, it would be interesting to analyse how $H_{C}$ would influence the procurement of DC grid reserves and how it could be combined with the instantaneous power limitation of generators (Equation (7)).

As a final remark, it has to be mentioned that wind power plants have limited capabilities to provide power services to DC grids due to their slow reaction time compared to DC grid time constants. However, if the DC grid is sufficiently damped by AC inertia or DC storage, the wind power plants can provide DCVCR. This can be achieved through a combined de-loading/de-rating control, which uses the fast de-loading reaction time and the de-rating capability of a permanent power change. Furthermore, DCVRR is technically feasible if the WPP is operated in de-rating mode. A problem could be a reliable forecast for the defined provision time. However, in both cases the available primary energy is not optimally used. Economic criteria will therefore ultimately decide whether wind power plants will contribute to DC reserves or whether another source might be more beneficial. 


\section{ACKNOWLEDGMENT}

The research leading to these results has received funding from the People Program (Marie Curie Actions) of the European Union's Seventh Framework Program FP7/2007-2013/ under REA grant agreement no. 317221, project title MEDOW.

\section{REFERENCES}

[1] "FERC Glossary." [Online]. Available: https://www.ferc.gov/ market-oversight;http://glossary.asp

[2] "EURELECTRIC Terminology." [Online]. Available: http://www. eurelectric.org/facts-terminology

[3] R. H. Renner and D. Van Hertem, "Ancillary Services in Electric Power Systems with HVDC Grids," IET Generation Transmission \& Distribution, vol. 9, no. 11, pp. 1179-1185, 2015.

[4] G. C. Tarnowski, P. C. Kjær, S. Dalsgaard, and A. Nyborg, "Regulation and frequency response service capability of modern wind power plants," in Power and Energy Society General Meeting (PESGM). IEEE, 2010, pp. $1-8$.

[5] R. Goic, D. Jakus, and J. Krstulovic, "Wind power plant as ancillary service provider," in 8th International Conference on the European Energy Market (EEM). IEEE, 2011, pp. 562-567.

[6] J. Van de Vyver, T. Vandoorn, J. De Kooning, B. Meersman, and L. Vandevelde, "Provision of Ancillary Services with Variable Speed Wind Turbines," in Proceedings IEEE young researchers symposium (YRS2014). EESA, 2014, pp. 1-6.

[7] B. Silva, C. Moreira, L. Seca, Y. Phulpin, and J. Lopes, "Provision of inertial and primary frequency control services using offshore multiterminal HVDC networks," IEEE Transactions on Sustainable Energy (STE), vol. 3, no. 4, pp. 800-808, 2012.

[8] L. Zeni, J. Glasdam, B. Hesselbæk, T. Lund, P. E. Sørensen, A. D Hansen, and P. C. Kjær, "Coordinated system services from offshore wind power plants connected through HVDC networks," 45th Session of the International Council on Large Electric Systems (CIGRE), 2014.

[9] P. Tielens and D. V. Hertem, "Receding Horizon Control of Wind Power to Provide Frequency Regulation," IEEE Transactions on Power Systems (PWRS), vol. 32, no. 4, Jul. 2017.

[10] J. Tan and Y. Zhang, "Coordinated Control Strategy of a Battery Energy Storage System to Support a Wind Power Plant Providing Multi-Timescale Frequency Ancillary Services," IEEE Transactions on Sustainable Energy (STE), vol. 8, no. 3, Jul. 2017.

[11] R. H. Renner and D. Van Hertem, "Potential of using DC voltage restoration reserve for HVDC grids," Electric Power Systems Research (EPSR), vol. 134, pp. 167-175, 2016.

[12] A. Egea, "Multiterminal HVDC transmissions systems for offshore wind," Ph.D. dissertation, Universitat Politècnica de Catalunya, Sep 2014.

[13] A.-K. Marten, "Operation of meshed high voltage direct current (HVDC) overlay grids," Ph.D. dissertation, Technischen Universität Ilmenau, 2015.

[14] Y. G. Rebours, D. S. Kirschen, M. Trotignon, and S. Rossignol, "A survey of frequency and voltage control ancillary services-Part I: Technical features," IEEE Transactions on Power Systems (PWRS), vol. 22, no. 1, pp. 350-357, 2007.

[15] P. Kundur, Power System Stability and Control . McGraw-Hill, Inc., 1994.

[16] A. Schwab, Elektroenergiesysteme, 2nd ed. Springer-Verlag, 2009.

[17] "DIgSILENT PowerFactory 15 User Manual," 2013.

[18] J. E. Normey-Rico, Control of dead-time processes. Springer Science \& Business Media, 2007.

[19] J. Beerten, "Modeling and Control of DC Grids," Ph.D. dissertation, KU Leuven, May 2013.
Table III: Generator data, [17].

\begin{tabular}{|c|c|c|}
\hline Apparent power & & 255 MVA \\
\hline Nominal voltage & & $19 \mathrm{kV}$ \\
\hline Inertia constant & & $5 \mathrm{~s}$ \\
\hline Number of generators & & 4 \\
\hline Synchronous reactances & $\begin{array}{l}x_{d} \\
x_{q}\end{array}$ & $\begin{array}{l}2.01 \mathrm{pu} \\
1.89 \mathrm{pu}\end{array}$ \\
\hline Transient reactances & $\begin{array}{l}x_{d}^{\prime} \\
x_{q}^{\prime}\end{array}$ & $\begin{array}{l}0.21 \mathrm{pu} \\
0.34 \mathrm{pu}\end{array}$ \\
\hline Subtransient reactances & $\begin{array}{l}x_{d}^{\prime \prime} \\
x_{q}^{\prime \prime}\end{array}$ & $\begin{array}{l}0.16 \mathrm{pu} \\
0.17 \mathrm{pu}\end{array}$ \\
\hline Transient time constants & $\begin{array}{l}t_{d}^{\prime} \\
t_{q}^{\prime}\end{array}$ & $\begin{array}{l}1.08 \mathrm{~s} \\
0.18 \mathrm{~s}\end{array}$ \\
\hline Subtransient time constants & $\begin{array}{l}t_{d}^{\prime \prime} \\
t_{q}^{\prime \prime}\end{array}$ & $\begin{array}{l}0.018 \mathrm{~s} \\
0.018 \mathrm{~s}\end{array}$ \\
\hline
\end{tabular}

Table IV: Data for automatic voltage regulation (AVR) IEEEX1 [17].

\begin{tabular}{c|c} 
Measurement delay & $0.02 \mathrm{~s}$ \\
\hline Controller gain & $200 \mathrm{pu}$ \\
\hline Controller time constant & $0.05 \mathrm{~s}$ \\
\hline Filter delay time & $1 \mathrm{~s}$ \\
\hline Filter derivative time constant & $1 \mathrm{~s}$ \\
\hline Exciter time constant & $0.05 \mathrm{~s}$ \\
\hline Stabilisation path gain & $0.04 \mathrm{pu}$ \\
\hline Stabilisation path delay time & $1 \mathrm{~s}$ \\
\hline Saturation factor 1 & $3.9 \mathrm{pu}$ \\
\hline Saturation factor 2 & $0.1 \mathrm{pu}$ \\
\hline Saturation factor 3 & $5.2 \mathrm{pu}$ \\
\hline Saturation factor 4 & $0.5 \mathrm{pu}$ \\
\hline Exciter constant & $1 \mathrm{pu}$ \\
\hline Controller minimum output & $-5 \mathrm{pu}$ \\
\hline Controller maximum output & $5 \mathrm{pu}$
\end{tabular}

Table V: Data for IEEEG1 governor model, [17].

\begin{tabular}{c|c} 
Controller gain & $10 \mathrm{pu}$ \\
\hline Governor time constant & $0.2 \mathrm{~s}$ \\
\hline Governor derivative time constant & $1 \mathrm{~s}$ \\
\hline Servo time constant & $0.6 \mathrm{~s}$ \\
\hline High pressure turbine factor & $0.3 \mathrm{pu}$ \\
\hline Intermediate pressure turbine time constant & $0.5 \mathrm{~s}$ \\
\hline Intermediate pressure turbine factor & $0.25 \mathrm{pu}$ \\
\hline Medium pressure turbine time constant & $0.8 \mathrm{~s}$ \\
\hline Medium pressure turbine factor & $0.3 \mathrm{pu}$ \\
\hline High pressure turbine time constant & $0.6 \mathrm{~s}$ \\
\hline Low pressure turbine time constant & $1.0 \mathrm{~s}$ \\
\hline Low pressure turbine factor & $0.15 \mathrm{pu}$ \\
\hline Valve closing time & $-0.3 \mathrm{pu} / \mathrm{s}$ \\
\hline Valve opening time & $0.3 \mathrm{pu} / \mathrm{s}$ \\
\hline Minimum gate limit & $0 \mathrm{pu}$ \\
\hline Maximum gate limit & $1 \mathrm{pu}$
\end{tabular}

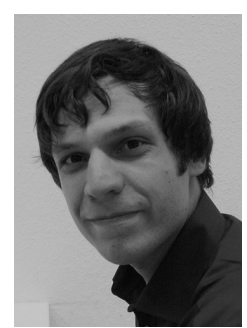

Robert H. Renner (S'09) received the Dipl.Ing. (FH) degree from University of Applied Sciences Bielefeld in 2010, the M.Sc. from Otto-vonGuericke-University Magdeburg in 2012 and the $\mathrm{Ph} . \mathrm{D}$. degree in electrical engineering from KU Leuven in 2017. His research interests are the operation of combined AC and DC grids and the integration of controllable devices such as voltage source converters (VSC) in the AC power system.

Robert H. Renner is also an active member of IEEE and Cigré. 


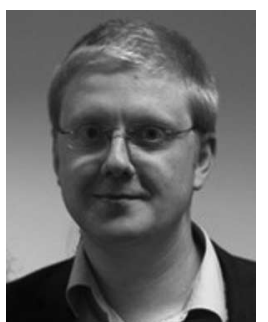

Dirk Van Hertem (S02SM09) received the M.Eng. degree from the Katholieke Hogeschool Kempen (KHK), Geel, Belgium, in 2001. He received the M.Sc. and the Ph.D. degree in electrical engineering from the University of Leuven, Leuven, Belgium, in respectively 2003 and 2009. In 2010, he was a Member of the Electric Power Systems (EPS) group with the Royal Institute of Technology, Stockholm, Sweden. Currently he is back at the University of Leuven, where he is an Associate Professor with the Electrical Energy and Computer Architectures (ELECTA) group. His research interests include power system operation and control in systems with FACTS and high-voltage direct current (HVDC) and building the transmission system of the future, including offshore grids and the supergrid concept.

Dr. Van Hertem is an Active Member of both IEEE (Power and Energy Society (PES) and Industry Applications Society (IAS)) and Cigré. 